\title{
Cognitive performance in irritable bowel syndrome: evidence of a stress-related impairment in visuospatial memory
}

\author{
P. J. Kennedy ${ }^{1,2}$, G. Clarke ${ }^{1,2}$, A. O’Neill ${ }^{1}$, J. A. Groeger ${ }^{3}$, E. M. M. Quigley ${ }^{1,4}$, F. Shanahan ${ }^{1,4}$, \\ J. F. Cryan ${ }^{1,5}$ and T. G. Dinan ${ }^{1,2 *}$ \\ ${ }^{1}$ Alimentary Pharmabiotic Centre, University College Cork, Ireland \\ ${ }^{2}$ Department of Psychiatry, University College Cork, Ireland \\ ${ }^{3}$ Department of Psychology, University of Hull, UK \\ ${ }^{4}$ Department of Medicine, University College Cork, Ireland \\ ${ }^{5}$ Department of Anatomy and Neuroscience, University College Cork, Ireland
}

Background. Central nervous system (CNS) dysfunction is a prominent feature of the functional gastrointestinal (GI) disorder, irritable bowel syndrome (IBS). However, the neurobiological and cognitive consequences of key pathophysiological features of IBS, such as stress-induced changes in hypothalamic-pituitary-adrenal (HPA)-axis functioning, is unknown. Our aim was to determine whether IBS is associated with cognitive impairment, independently of psychiatric co-morbidity, and whether cognitive performance is related to HPA-axis function.

Method. A cross-sectional sample of 39 patients with IBS, a disease control group of 18 patients with Crohn's disease (CD) in clinical remission and 40 healthy age- and IQ-matched control participants were assessed using the Paired Associates Learning (PAL), Intra-Extra Dimensional Set Shift (IED) and Spatial Working Memory (SWM) tests from the Cambridge Neuropsychological Test Automated Battery (CANTAB) and a computerized Stroop test. HPA-axis function was determined by measuring the cortisol awakening response (CAR).

Results. IBS patients exhibited a subtle visuospatial memory deficit at the PAL six- pattern stage $(p=0.03)$, which remained after psychiatric co-morbidity was controlled for $(p=0.04)$. Morning cortisol levels were lower in IBS $(p=0.04)$ and significantly associated with visuospatial memory performance within IBS only $(p=0.02)$.

Conclusions. For the first time, altered cognitive function on a hippocampal-mediated test of visuospatial memory, which was related to cortisol levels and independent of psychiatric co-morbidity, has been identified in IBS. Visuospatial memory impairment may be a common, but currently neglected, component of IBS. Further elucidation of the nature of this impairment may lead to a greater understanding of the underlying pathophysiology of IBS, and may provide novel therapeutic approaches.

Received 18 April 2013; Revised 30 July 2013; Accepted 4 August 2013; First published online 29 August 2013

Key words: CANTAB, cognition, cortisol, irritable bowel syndrome (IBS), stress.

\section{Introduction}

Irritable bowel syndrome (IBS) is the most common functional gastrointestinal (GI) disorder in Western countries, affecting an estimated $10 \%$ of the general population (Clarke et al. 2009b) and accounting for up to $50 \%$ of all visits to general practitioners for GI complaints (Wilson et al. 2004). Symptoms, which include abdominal pain or discomfort and altered bowel habits (Longstreth et al. 2006), are chronic (Ford et al. 2008) in some cases debilitating, and are

\footnotetext{
* Address for correspondence: Professor T. G. Dinan, M.D., D.Sc. Ph.D., Department of Psychiatry, GF Unit, Cork University Hospital, Wilton, Cork, Ireland.

(Email: t.dinan@ucc.ie)
}

cited as a leading cause of work absenteeism and presenteeism (Spiegel, 2009). IBS is thus associated with a substantial impairment in the individual sufferer's quality of life (Longstreth et al. 2003).

Although the pathophysiology of IBS is not fully understood, it is best regarded as a disorder caused by dysregulation of the complex interactions along the brain-gut axis (Cryan \& O'Mahony, 2011; Grenham et al. 2011; Mayer \& Tillisch, 2011; Collins et al. 2012), a model describing the bidirectional communication between the central, autonomic and enteric nervous systems, involving neural, endocrine and immune pathways (Ohman \& Simren, 2007). For example, it is thought that stress-induced changes in hypothalamic-pituitary-adrenal (HPA)-axis functioning can dysregulate normal brain-gut interactions 
(Mayer, 2000) and studies from our laboratory and others have documented abnormal HPA-axis activity in IBS (Bohmelt et al. 2005; Dinan et al. 2006; Chang et al. 2009). A role for low-grade immune activity in IBS has garnered much support from experimental studies demonstrating alterations on several immune parameters (Barbara et al. 2004; Collins, 2005; Guilarte et al. 2007; Ohman \& Simren, 2010), including elevated peripheral levels of pro-inflammatory cytokines (Dinan et al. 2006, 2008; Liebregts et al. 2007; Scully et al. 2010; McKernan et al. 2011) and an immunemediated degradation of tryptophan metabolism along the kynurenine pathway (Fitzgerald et al. 2008; Clarke et al. 2009a, 2012). Moreover, central dysfunction is a prominent feature of IBS. Centrally acting therapies, both psychotherapeutic (Whorwell et al. 1984, 1987; Lackner et al. 2006) and pharmacological (Creed et al. 2003), have demonstrated efficacy in the treatment of GI symptoms in IBS. Studies commonly report an increased prevalence of depression and anxiety, and affected patients often have a formal, psychiatrically diagnosed mood disorder (Hazlett-Stevens et al. 2003; Creed et al. 2005, 2008; Lembo et al. 2009; Mykletun et al. 2010). In addition, mounting evidence from functional magnetic resonance imaging (fMRI) research indicates that, in response to visceral pain stimulation, patients with IBS exhibit abnormal brain activity in regions involved in pain processing and endogenous pain modulation (Mayer et al. 2009; Tillisch et al. 2011). However, a largely understudied and potentially underestimated manifestation of brain-gut axis dysfunction in IBS is cognitive impairment (Kennedy et al. 2012).

To date, the few studies assessing cognitive performance in IBS are in line with a cognitive behavioural framework (Sharpe et al. 1992; Creed, 2007; Spence \& Moss-Morris, 2007) by demonstrating that patients exhibit attentional biases to GI symptom-related stimuli (Gibbs-Gallagher et al. 2001; Afzal et al. 2006; Posserud et al. 2009; Martin \& Chapman, 2010; Chapman \& Martin, 2011) or negatively valenced words (Gomborone et al. 1993). However, we recently proposed a cognitive neurobiological model whereby some of the key pathophysiological features of IBS, including stress, immune activation and chronic pain, may impact not only on emotion- or symptom-related cognition but also on key domains of executive function, working memory, attention and episodic memory (Kennedy et al. 2012). This model has received support from an fMRI study reporting impaired cognitive flexibility on the Wisconsin Card Sorting Test (WCST), with associated differences in regional activity in frontal and temporal brain regions in IBS (Aizawa et al. 2012). Although this is an important and substantial step in elucidating the cognitive domains that are affected in
IBS, it is limited in its scope of assessment by focusing on executive function alone. As such, the impact on other key brain functions such as episodic and working memory or non-emotional/symptom-related attention in IBS remains unknown. Furthermore, there remains a lack of understanding of how key pathophysiological features of IBS, such as stress-induced changes in HPA-axis functioning (Dinan et al. 2006), can impact on the brain and lead to cognitive impairment. Considering the prevalence of IBS and the high incidence among young adult females who may be engaged in formal education or embarking on careers, it is of great importance to explore the extent of cognitive impairment in this debilitating functional GI disorder.

The current study aimed to expand on previous findings by assessing how IBS patients perform on a battery of cognitive tests covering cognitive flexibility and attentional set-shifting (Intra-Extra Dimensional Set Shift; IED), working memory (Spatial Working Memory; SMW) and visuospatial episodic memory (Paired Associates Learning; PAL) from the Cambridge Neuropsychological Test Automated Battery (CANTAB $\left.{ }^{\circ}\right)$, and response conflict using the Stroop test. Considering that IBS is a stress-related disorder, our primary hypothesis was that, in addition to impaired cognitive flexibility as described previously (Aizawa et al. 2012), patients with IBS would exhibit mild but identifiable hippocampal-mediated, visuospatial memory dysfunction, which would be related to HPA-axis functioning as measured by the cortisol awakening response (CAR). In addition, we included the SWM and Stroop tests based on the hypothesis that impaired working memory and inhibitory responding have been identified in other conditions associated with stress and chronic pain, such as fybromyalgia and chronic fatigue syndrome (Glass, 2006; Moriarty et al. 2011), and thus performance on these tests may also be compromised in IBS. We compared cognitive performance in IBS with that of a group of individuals with a well-defined inflammatory GI disorder, Crohn's disease (CD). These patients were included as our disease control group to control for the effects of chronic GI symptoms. Additionally, we explicitly controlled for any possible impact of anxiety and depression on cognitive performance by carrying out a reanalysis of the data where cognitive differences were identified, following the removal of participants exhibiting clinically relevant levels of psychiatric co-morbidity.

\section{Method}

\section{Participants}

Patients with IBS who satisfied Rome III criteria (Longstreth et al. 2006) and had undergone previous 
investigation to exclude the presence of organic GI disease, including inflammatory bowel disease (IBD) and coeliac disease, and patients with $\mathrm{CD}$ in clinical remission [Harvey Bradshaw Index (HBI) score <5] were recruited from speciality clinics at Cork University Hospital. Healthy control participants were recruited from the staff and student population of University College Cork by advertisement. Patients presenting with significant acute or chronic coexisting illness other than that under study were excluded. Study participants were males and females between 18 and 50 years of age. Exclusion criteria included use of psychoactive medications (anxiolytics, antipsychotics, antidepressants at a dose used to treat depressive disorders, and opioid-based pain relievers), regular use of non-steroidal anti-inflammatory drugs (NSAIDs), antibiotic use within the prior 4 weeks, history of alcohol abuse, evidence of immunodeficiency and recent (within 6 months) abdominal surgery. Patients with $C D$ were required to have at least a 1-month wash-out period from prednisone use prior to testing. Budesonide, which has minimal systemic bioavailability, was allowed. Healthy control participants were excluded if they had a history of chronic complaints, GI (not attributable to gastroenteritis or other clearly identifiable cause) or otherwise.

\section{Study procedures}

The study protocol (APC024 2010) and all procedures were approved by the University College Cork Clinical Research Ethics Committee of the Cork Teaching Hospitals and conducted in accordance with the International Conference on Harmonization (ICH) Good Clinical Practice (GCP) Guidelines and the Declaration of Helsinki. Study participants meeting inclusion criteria for entry provided written informed consent before participation. A total of 97 individuals enrolled in the study. Groups were matched on the basis of age, verbal IQ, body mass index (BMI) and units of alcohol consumed per week. Study visits were conducted between 0730 and $1030 \mathrm{~h}$ to control for endogenous fluctuations in glucocorticoid levels. On arrival at the laboratory, a brief medical examination was carried out by an experienced clinical research nurse who measured participants' vital signs, recorded their BMI and collected a venous blood sample for assessment of full blood count, renal function, serum electrolytes and liver enzymes. Clinically significant abnormalities in these blood tests led to exclusion from the study following review by an experienced physician.

\section{Measures}

\section{Mood and GI symptom questionnaires}

Symptoms of anxiety and depression were assessed using the self-reported Hospital Anxiety and Depression Scale (HADS; Zigmond \& Snaith, 1983) and the nine-item Patient Health Questionnaire (PHQ-9; Kroenke et al. 2001). Patients with IBS rated their current abdominal pain severity on a scale from 0 (no pain) to 100 (severe pain). CD patients completed the $\mathrm{HBI}$ as a general index of current symptomatology.

\section{Salivary cortisol analysis}

HPA-axis functioning was examined by measuring the CAR. This is a well-validated method for characterizing HPA-axis function and has been used for this purpose in numerous functional and psychiatric disorders (Fries et al. 2009). On the morning prior to their study visit, participants were instructed to collect saliva samples upon wakening, and at $1 \mathrm{~h}$ and $3 \mathrm{~h}$ postwakening. As it has not yet been established whether variable waking times affect the CAR (for reviews, see Fries et al. 2009; Law et al. 2013), we took the approach that is common in the literature (Hinkelmann et al. 2013) and did not require that participants wake at a specific time, but followed their normal routine as closely as possible. Waking times were recorded for analysis to determine any group differences. Salivary samples were stored at $-80^{\circ} \mathrm{C}$ until analysis. Cortisol concentrations were determined using the Cortisol Enzyme Immunoassay Kit according to the manufacturer's instruction $\left(\right.$ Enzo $^{\circledR}$ Life Sciences, UK). The assay detection limit was $0.16 \mathrm{nmol} / \mathrm{l}$. Inter- and intra-assay coefficients of variation $(\mathrm{CVs})$ were $11.24 \%$ and $8.2 \%$ respectively.

\section{Cognitive assessment}

Tests from the CANTAB ${ }^{\circledR}$ battery (Cambridge Cognition, Ltd, UK; Robbins \& Sahakian, 1994) and a computerized Stroop word-colour interference test (Stroop; Xavier Educational Software Ltd, UK) were administered by a trained test administrator who issued standardized verbal instructions to participants on the use of a portable touch screen Sahara i440D Slate Tablet PC (TabletKiosk, Sand Dune Ventures, USA) running CANTABeclipse ${ }^{\mathrm{TM}}$ software. The cognitive assessment lasted approximately $45 \mathrm{~min}$ with each participant first completing the Big/Little Circle as a short familiarization task, followed by the IED, PAL and SWM tests from the CANTAB, and finally the Stroop test. A measure of pre-morbid IQ was obtained using the National Adult Reading Test-2 (NART-2; Nelson \& Willison, 1991) and converted to Wechsler Adult Intelligence Scale-Revised (WAIS-R) full-scale 
IQ scores. A brief description of each cognitive test is provided in the following sections. More detailed information on CANTAB tests is available elsewhere (Sahakian \& Owen, 1992; Fray \& Robbins, 1996).

\section{$P A L$, parallel mode}

PAL is a test of visuospatial episodic memory and assesses new learning, list memory and list learning, and has demonstrated sensitivity to changes in the function of hippocampal brain regions (Owen et al. 1995; Sweeney et al. 2000; Swainson et al. 2001; Blackwell et al. 2004). However, performance on PAL has also been shown to engage additional brain regions comprising the frontoparietal network during encoding phases, and posterior cingulate and left cuneus regions during retrieval stages (de Rover et al. 2011). Outcome measures assessed were errors made at the six-pattern and eight-pattern stage (adjusted), total errors (adjusted), mean trials to success, and first trial memory score.

\section{Stroop test}

The Stroop is a test of executive function and assesses selective attention and response inhibition. Inhibition of the prepotent response in the interference stage of the Stroop primarily engages the anterior cingulate cortex (ACC), with general Stroop performance also requiring input from regions of the temporal and parietal lobes (Botvinick et al. 2004; Alvarez \& Emory, 2006; Strauss et al. 2006). The computerized Stroop test used in the current study is based on the Victoria Stroop Test (VST) as described previously (Assef et al. 2007). Response speed in milliseconds is recorded on each trial with an overall mean response time (MRT) for each of three stages [Stage 1, word naming; Stage 2, colour naming; Stage 3 (interference stage), naming the colour of an incongruent word, for example the word 'blue' printed in the colour red], consisting of 24 trials. The main outcome measure is the 'Stroop effect', calculated by subtracting the MRT of Stage 1 from the MRT on Stage 3 (Assef et al. 2007).

\section{IED}

IED is a test of executive function and assesses rule acquisition and reversal, attentional set formation, maintenance and shifting (Downes et al. 1989; Sahakian \& Owen, 1992). Reversal learning has been shown to involve the ventral prefrontal cortex (PFC) whereas the dorsolateral PFC is engaged during attentional set-shifting (Nagahama et al. 2001). The outcome measures assessed were reversal learning (errors made on stages 2, 5, 7 and 9) and attentional flexibility (errors made on stages 6 and 8), as described previously (Mehta et al. 1999; Rahman et al. 1999), and total errors (adjusted).

SWM

SWM is a test of working memory, involving online monitoring and updating of information and selfordered searching, and has shown sensitivity to frontal lobe dysfunction (Owen et al. 1996; Robbins et al. 1998). The outcome measures assessed were total betweensearch errors, total errors and strategy score.

\section{Statistical analysis}

As the CANTAB variables were not normally distributed, the following transformations were applied. IED and PAL outcome variables were normalized using $\log _{10}$ transformations and SWM outcome variables were normalized using square-root transformations. The Stroop was completed by 38 controls, 38 IBS patients and $18 \mathrm{CD}$ patients, and data were analysed excluding the three participants who did not complete the test. The $\chi^{2}$ test was used to examine the gender distribution across groups. A one-way ANOVA was used to explore differences in participant waking times, group characteristics (age, IQ, BMI, units of alcohol per week), HADS-anxiety (HADS-A), HADS-depression (HADS-D) and PHQ-9 scores, and to assess individual measures of the PAL, IED, SWM and Stroop tests, followed by Tukey's Honestly Significant Difference (HSD) post-hoc test or Dunnett's $t$ contrasts, as appropriate. As this was an exploratory study, we have not specified a single study end-point and did not adjust for multiple end-points in a full factorial model (Bender \& Lange, 2001; Feise, 2002) to allow detection of possible differences in cognitive performance on each test within our study population. Total cortisol levels across each collection time point were determined using an area under the curve with respect to ground $\left(\mathrm{AUC}_{\mathrm{g}}\right)$ calculation (Pruessner et al. 2003). AUC $_{g}$ cortisol levels were transformed using a natural $\log (\ln )$ to correct positive skew and improve homogeneity of variance, followed by a one-way ANOVA and Dunnett's $t$ contrasts to identify patient versus control differences. Saliva samples were provided by 32 healthy controls, 36 IBS patients and $17 \mathrm{CD}$ patients as instructed and useable for analysis. Correlation analyses were carried out using Spearman's $\rho$ within each group (control/IBS/CD) to examine the influence of total morning cortisol levels $\left(\mathrm{AUC}_{\mathrm{g}}\right), \mathrm{IQ}$, anxiety (HADS-A), depression (HADS-D; PHQ-9), duration of disease, current abdominal pain severity in patients with IBS, and HBI total scores in $\mathrm{CD}$ patients on cognitive performance. Finally, as an additional measure to control for the influence of levels of anxiety or depression on cognitive performance, 
Table 1. Comparison of group demographics and clinical characteristics

\begin{tabular}{lllll}
\hline & $\begin{array}{l}\text { Healthy controls } \\
(n=40)\end{array}$ & IBS $(n=39)$ & CD $(n=18)$ & $p$ value \\
\hline $\begin{array}{l}\text { Age (years) } \\
\text { Gender }\end{array}$ & $28.3 \pm 1.43$ & $28.21 \pm 1.19$ & $32.67 \pm 2.11$ & 0.14 \\
$\quad$ Male & $11(27.5)$ & $6(15.4)$ & $11(61.1)$ & \\
Female & $29(72.5)$ & $33(84.6)$ & $7(38.9)$ & 0.002 \\
BMI (kg/m ${ }^{2}$ ) & $23.55 \pm 0.59$ & $23.74 \pm 0.63$ & $25.23 \pm 0.73$ & 0.23 \\
WAIS-R full-scale IQ (NART conversion) & $108.57 \pm 1.07$ & $105.48 \pm 1.39$ & $103.39 \pm 2.54$ & 0.07 \\
HADS-D & $0.85 \pm 0.15$ & $3.85 \pm 0.46$ & $2.99 \pm 0.70$ & $<0.001$ \\
HADS-A & $4.30 \pm 0.4$ & $8.21 \pm 0.74$ & $6.67 \pm 3.45$ & $<0.001$ \\
$\quad$ PHQ-9 & $2.03 \pm 0.36$ & $6.1 \pm 0.86$ & $3.89 \pm 0.94$ & $<0.001$ \\
Disease duration (years) & - & $9.13 \pm 1.13$ & $9.72 \pm 1.63$ & - \\
Current pain, abdominal pain severity (0-100) & - & $34.75 \pm 4.65$ & - & $1.89 \pm 0.31$ \\
HBI total score (remission<5) & - & - & & \\
\hline
\end{tabular}

IBS, Irritable bowel syndrome; CD, Crohn's disease; BMI, body mass index; WAIS-R, Wechsler Adult Intelligence Scale Revised; NART, National Adult Reading Test; HADS-A/D, Hospital Anxiety and Depression Scale - Anxiety/ Depression; PHQ-9, nine-item Patient Health Questionnaire; HBI, Harvey Bradshaw Index.

Data are given as $n(\%)$ or mean \pm standard error of the mean (S.E.M.).

participants meeting predefined criteria for possible co-morbid depression (a PHQ-9 score of $\geqslant 10$; Kroenke et al. 2001) and/or co-morbid anxiety (a HADS-A score of $\geqslant 11$; Snaith, 2003) were excluded, and a one-way ANOVA along with Tukey comparisons and Dunnett's $t$ contrasts was carried out on CANTAB and Stroop outcomes, where group differences had been identified with all participants included. Sample size was determined using a power calculation to detect significant group differences at an $\alpha$ level of 0.05 . The study was not powered to detect subgroup differences among IBS patients, although this information was recorded. Non-transformed data are presented as mean \pm standard error of the mean (s.E.M.). Effect sizes are reported as partial eta squared $\left(\eta_{\mathrm{p}}^{2}\right)$. All statistical procedures were carried out using SPSS version 20.0 (SPSS Inc., USA).

\section{Results}

\section{Sample characteristics}

Group characteristics for healthy control participants, IBS patients and CD patients are presented in Table 1. Groups did not differ significantly on age, IQ, BMI or number of units of alcohol consumed per week. One patient with $\mathrm{CD}$ was using budesonide (Entocort EC) at the time of testing. According to Rome III criteria, seven IBS patients were diarrhoea predominant (IBS-D), four constipation predominant (IBS-C) and 28 were mixed (IBS-M). Patients with IBS $(p<0.001)$ and patients with CD $(p=0.02)$ scored higher on the HADS-D than the healthy controls. Patients with IBS scored significantly higher on the PHQ-9 depression scale $(p<0.001)$ and the HADS-A $(p<0.001)$ than the healthy controls.

\section{Cognitive performance}

Visuospatial episodic memory (PAL)

Comparisons of the number of errors committed at each individual stage showed that the six-pattern stage $\left(F_{2,94}=3.43, p=0.03, \eta_{\mathrm{p}}^{2}=0.07\right.$; Fig. $\left.1 a\right)$, but not the eight-pattern stage $\left(F_{2,94}=0.02, p=0.98\right.$; Fig. $\left.1 b\right)$, differentiated patients from healthy controls. Post-hoc exploration of this group effect at the six-pattern stage using Tukey comparisons found that patients with IBS exhibited significantly $(p=0.03)$ impaired visuospatial memory performance compared to controls but not to patients with CD $(p=0.97)$. Patients with $\mathrm{CD}$ did not differ from controls in the number of errors made at the six-pattern stage $(p=0.2)$. There was no overall effect of group on total errors $\left(F_{2,94}=0.46, p=0.63, \eta_{\mathrm{p}}^{2}=0.01\right.$; Fig. $\left.1 c\right)$, mean number of trails to success $\left(F_{2,94}=1.05, \quad p=0.35, \quad \eta_{\mathrm{p}}^{2}=0.02\right.$; Fig. $1 d)$ or first trial memory score $\left(F_{2,94}=1.17\right.$, $p=0.32, \eta_{\mathrm{p}}^{2}=0.02$; Fig. $1 e$ ).

\section{Selective attention and response inhibition (Stroop)}

Group differences on the Stroop effect approached significance $\left(F_{2,91}=3.04, p=0.052, \eta_{\mathrm{p}}^{2}=0.06\right)$. Inspection of post-hoc Tukey comparisons showed that patients with CD $(p=0.04)$, but not patients with IBS $(p=0.9)$, 
Table 2. Summary of mean test scores on the Stroop, IED and SWM

\begin{tabular}{llll}
\hline Cognitive test & Control & IBS & CD \\
\hline Stroop effect (ms) & $297.55 \pm 29.22$ & $320.31 \pm 38.96$ & $463.89 \pm 81.52^{*}$ \\
IED & & & $8.44 \pm 0.2$ \\
$\quad$ Stages complete & $8.5 \pm 0.14$ & $8.5 \pm 0.14$ & $27.17 \pm 4.67$ \\
$\quad$ Total errors (adjusted) & $24.67 \pm 3.23$ & $24.51 \pm 3.25$ & $7.67 \pm 2.17$ \\
Reversal learning (errors) & $5.05 \pm 0.44$ & $6.23 \pm 0.92$ & $11.89 \pm 2.43$ \\
Attentional flexibility (errors) & $11.92 \pm 1.72$ & $10.79 \pm 1.54$ & \\
SWM & & & $25.44 \pm 4.62$ \\
Total between errors & $18.57 \pm 2.81$ & $23.43 \pm 2.57$ & $26.5 \pm 4.81$ \\
Total errors & $19.4 \pm 3.02$ & $24.15 \pm 2.68$ & $29.78 \pm 1.63$ \\
Strategy score & $30.25 \pm 1.04$ & $31.9 \pm 0.99$ & \\
\hline
\end{tabular}

IED, Intra-Extra Dimensional Set Shift; SWM, Spatial Working Memory; IBS, irritable bowel syndrome; CD, Crohn's disease.

${ }^{*} p<0.05 v$. control.

Data are given as mean \pm standard error of the mean (S.E.M.).

(a)

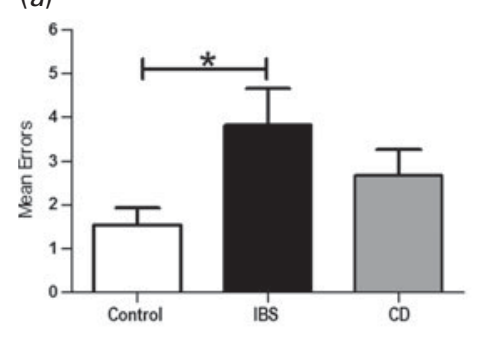

(b)

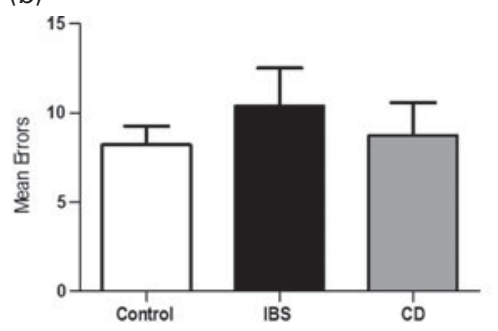

(c)

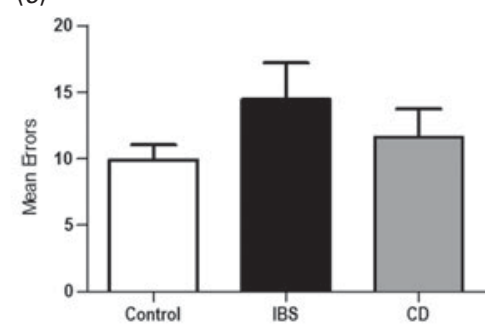

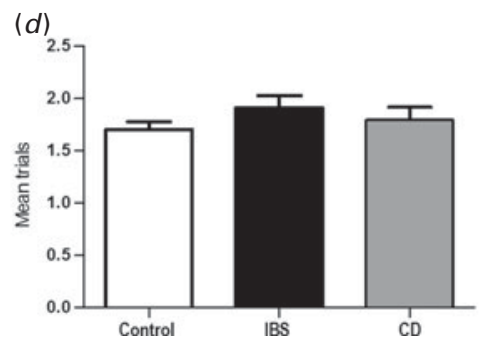

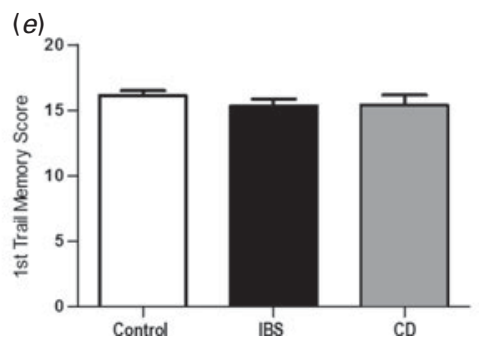

Fig. 1. Group comparison of visuospatial episodic memory performance on the Paired Associates Learning (PAL) test. (a) Mean errors at the six-pattern stage [* $p<0.05$, irritable bowel syndrome (IBS) versus control]; (b) mean errors at the eight-pattern stage; $(c)$ mean total errors; $(d)$ average trials needed to successfully complete each stage; $(e)$ average number of correct choices on the first trial of each stage; lower score=poorer performance. $\mathrm{CD}$, Crohn's disease. Data are presented as mean \pm standard error of the mean (S.E.M.).

were significantly less able to cope with Stroop interference than healthy controls (Table 2).

\section{Executive function (IED)}

Groups did not differ significantly on total stages complete $\left(F_{2,94}=0.04, p=0.9, \eta_{\mathrm{p}}^{2}=0.001\right)$, measures of reversal learning $\left(F_{2,94}=0.68, p=0.51, \eta_{p}^{2}=0.01\right)$, attentional flexibility $\left(F_{2,94}=0.053, p=0.95, \eta_{\mathrm{p}}^{2}=0.001\right)$ or the total number of errors across all stages of the IED test
$\left(F_{2,94}=0.16, p=0.85, \eta_{\mathrm{p}}^{2}=0.003\right.$; see Table 2 for mean scores).

\section{SWM}

No significant main effect of group was found for the SWM strategy test $\left(F_{2,94}=0.92, p=0.4, \eta_{\mathrm{p}}^{2}=0.02\right)$, total between errors $\left(F_{2,94}=1.45 ; p=0.24, \eta_{\mathrm{p}}^{2}=0.03\right)$ or total errors across each stage $\left(F_{2,94}=1\right.$. 36; $p=0.26, \eta_{\mathrm{p}}^{2}=0.02$, see Table 2 for mean scores). 


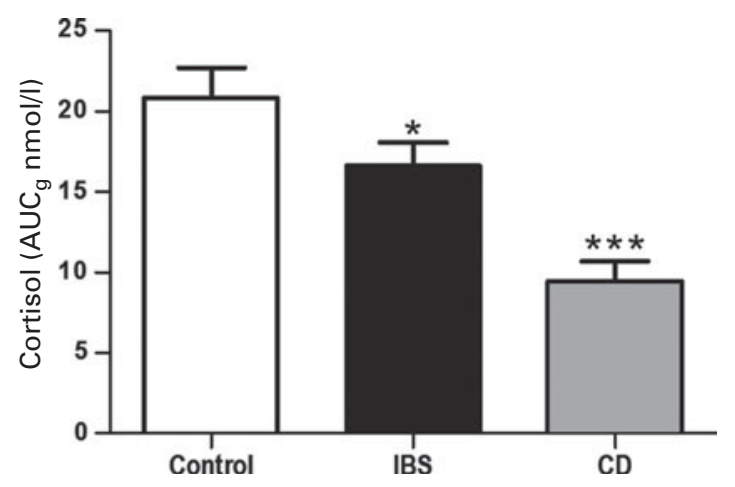

Fig. 2. Group comparison of total morning cortisol levels determined using an area under the curve with respect to ground $\left(\mathrm{AUC}_{\mathrm{g}}\right)$ calculation on all three measurement points in healthy control participants $(n=34)$, patients with irritable bowel syndrome (IBS; $n=36$ ) and patients with Crohn's disease $(\mathrm{CD} ; n=17){ }^{*} p<0.05,{ }^{* * *} p<0.001$ versus control. Data are presented as mean \pm standard error of the mean (S.E.M.).

\section{Morning cortisol levels}

Waking times for healthy controls (mean $=0813$ $\mathrm{h} \pm 13 \mathrm{~min}$ ), patients with IBS (mean $=0820 \mathrm{~h} \pm 14 \mathrm{~min}$ ) and patients with $\mathrm{CD}$ (mean $=0808 \mathrm{~h} \pm 26 \mathrm{~min}$ ) were not significantly different $\left(F_{2,82}=0.12, p=0.89\right)$. Total morning cortisol levels $\left(\mathrm{AUC}_{\mathrm{g}}\right)$ differed significantly between patients and controls $\left(F_{2,82}=13.71, p<0.001\right.$, $\eta_{\mathrm{p}}^{2}=0.25$; Fig. 2). Post-hoc analysis using Dunnett's $t$ contrasts against controls showed that patients with IBS $(p=0.03)$ and patients with CD $(p<0.001)$ exhibited lower total cortisol levels across the three measurement time points in comparison to healthy controls.

\section{Correlation analysis}

A correlation analysis was carried out to identify relationships between visuospatial memory performance (errors at the PAL six-pattern stage), selective attention and response inhibition (the Stroop test), total morning cortisol levels (AUC $\mathrm{g}$ ), IQ (WAIS-R), levels of anxiety (HADS-A) and levels of depression (HADS-D, PHQ-9). A significant negative relationship was identified between $\mathrm{AUC}_{\mathrm{g}}$ and visuospatial memory performance within the IBS group but not in the healthy control participants or the patients with CD (Table 3). Additional correlations were carried out within the patient groups to examine the relationship between disease duration, current abdominal pain (IBS patients) and HBI (CD patients), and cognitive performance. Within the IBS group, no significant relationships were identified between disease duration or current abdominal pain and PAL six-pattern errors (both $p>0.05$ ). Similarly, within the CD group, no significant relationship was identified between disease duration or HBI scores and Stroop performance (both $p>0.05)$.

\section{Cognitive performance after controlling for psychiatric co-morbidity}

To further control for any influence of levels of depression and anxiety on cognitive performance, participants meeting the predefined criteria for psychiatric co-morbidity (PHQ-9 score of $\geqslant 10$; Kroenke et al. 2001) and/or co-morbid anxiety (HADS-A score of $\geqslant 11$; Snaith, 2003) were excluded and we reanalysed the number of errors made at the PAL six-pattern stage and the performance on the Stroop interference test. The predefined criteria for psychiatric co-morbidity were met by $16(41.02 \%)$ of the IBS patients and five $(27.78 \%)$ of the CD patients but none of the control participants. Reanalysis using ANOVA showed that group differences on visuospatial episodic memory performance (errors at PAL six-pattern stage; control: $n=40$, IBS: $n=23, \mathrm{CD}: n=13)$ remained significant $\left(F_{2,73}=3.39, p=0.03, \eta_{\mathrm{p}}^{2}=0.08\right)$. Post-hoc analysis using Tukey comparisons showed that patients with IBS and without psychiatric co-morbidity made significantly more errors at the PAL six-pattern stage than healthy controls $(p=0.04)$. Overall, group differences on selective attention and response inhibition (Stroop interference effect; control: $n=38$, IBS: $n=22$, CD: $n=13)$, after controlling for psychiatric comorbidity, did not reach significance $\left(F_{2,70}=2.11 ; p=\right.$ $\left.0.129, \eta_{\mathrm{p}}^{2}=0.57\right)$. However, post-hoc inspection of Dunnett's $t$ contrasts showed that patients with CD without psychiatric co- morbidity, when compared to healthy control participants, still exhibited reduced attentional capacity, as they continued to be significantly less able to manage Stroop interference than controls $(p=0.04)$.

\section{Discussion}

In this study we tested the hypothesis that IBS is associated with cognitive impairment. We used CANTAB, a highly standardized and well-validated cognitive test battery (Strauss et al. 2006), to assess performance in IBS and CD in comparison with healthy controls across several cognitive domains including reversal learning and attentional flexibility (IED), selective attention and response inhibition (Stroop), working memory (SWM) and visuospatial episodic memory (PAL). To our knowledge, this is the first time that such a detailed cognitive assessment has been performed in either condition.

In support of our main hypothesis, we have shown that patients with IBS exhibit a subtle deficit in visuospatial episodic memory functioning, which was clear 
Table 3. Summary of correlations between cognitive performance (errors at the PAL six-pattern stage and the Stroop test) and morning cortisol levels, IQ and clinical characteristics

\begin{tabular}{|c|c|c|c|c|c|c|}
\hline \multirow[b]{2}{*}{ Measure } & \multicolumn{3}{|c|}{ Errors PAL six-pattern stage } & \multicolumn{3}{|c|}{ Stroop test } \\
\hline & Control & IBS & $\mathrm{CD}$ & Control & IBS & $\mathrm{CD}$ \\
\hline Morning cortisol $\left(\mathrm{AUC}_{\mathrm{g}}\right)$ & 0.023 & $-0.367^{*}$ & -0.437 & -0.005 & -0.013 & 0.032 \\
\hline WAIS-R IQ & -0.237 & -0.121 & -0.035 & 0.080 & -0.113 & 0.266 \\
\hline HADS-A (Anxiety) & 0.114 & 0.008 & -0.047 & 0.130 & -0.142 & 0.317 \\
\hline HADS-D (Depression) & 0.005 & 0.066 & 0.361 & 0.237 & -0.107 & 0.351 \\
\hline PHQ-9 (Depression) & -0.013 & 0.025 & -0.038 & 0.089 & 0.072 & 0.284 \\
\hline Disease duration & - & 0.18 & 0.16 & - & -0.01 & 0.37 \\
\hline Current abdominal pain & - & -0.27 & - & - & -0.02 & - \\
\hline HBI & - & - & -0.07 & - & - & -0.20 \\
\hline
\end{tabular}

PAL, Paired Associate Learning; WAIS-R, Wechsler Adult Intelligence Scale-Revised; HADS-A/D, Hospital Anxiety and Depression Scale - Anxiety/Depression; PHQ-9, nine-item Patient Health Questionnaire; HBI, Harvey Bradshaw Index. ${ }^{*} p<0.05$.

at the six-pattern stage of PAL. Performance on PAL is highly dependent on the functioning of the hippocampus (de Rover et al. 2011), a brain region that is intrinsically involved in the negative feedback regulation of the HPA axis and where glucocorticoid receptors are highly expressed (McEwen, 1999). In our sample, patients with IBS exhibited lower basal morning cortisol levels in comparison to healthy controls, a finding that is in support of some (Bohmelt et al. 2005; Suarez-Hitz et al. 2012), but not all (Chang et al. 2009), previous reports. Nonetheless, these results add to the well-acknowledged view that HPA-axis dysfunction is a key feature of IBS (Mayer et al. 2001). Furthermore, our finding of a relationship between morning cortisol levels and performance on the PAL test is in line with many preclinical (Song et al. 2006; Sterlemann et al. 2010) and human studies (Lupien et al. 2009) that have documented the negative impact of HPA-axis dysregulation on hippocampal-mediated cognitive performance. Our results suggest that lower cortisol levels are associated with increased PAL errors in IBS, and this is in line with the commonly acknowledged inverted U-shape function of cortisol on hippocampal-mediated memory performance, where both abnormally elevated and blunted cortisol levels are associated with cognitive dysfunction (de Kloet $e t$ al. 1999; Wolf, 2003; Lupien et al. 2007). Indeed, previous cognitive assessments in IBS (Gomborone et al. 1993; Gibbs-Gallagher et al. 2001; Afzal et al. 2006; Posserud et al. 2009; Martin \& Chapman, 2010; Chapman \& Martin, 2011), together with our findings, suggest that IBS may be associated with both emotionally modulated cognitive alterations mediated by amygdalar regions and non-emotional visuospatial episodic memory alterations mediated by the hippocampus.
Brain imaging studies have suggested a key role for the ACC in abnormal central processing of visceral pain in IBS (Mayer et al. 2009). Patients with IBS in our sample did not exhibit deficits on the Stroop test, which heavily engages the ACC during the interference stages (Alvarez \& Emory, 2006), suggesting that the ACC is unaffected at the cognitive level and that abnormal functioning of this region in IBS is specific to altered visceral pain processing. However, subregions of the ACC (the perigenual ACC and anterior midcingulate cortex) may be functioning differentially in IBS (Tillisch et al. 2011) and our findings may simply reflect that the Stroop does not fully engage these particular regions. Furthermore, it should be noted that altered visceral pain processing may be apparent in only a subset of patients with IBS (Quigley, 2006) and, as such, it is possible that only a subset of patients, not represented in our sample, may exhibit ACC-related cognitive deficits.

Altered cognitive flexibility on the WCST, accompanied by reduced right dorsolateral PFC activity, has recently been described in IBS (Aizawa et al. 2012). By contrast, we found no deficit in reversal learning or cognitive flexibility on the IED, which is considered to be an analogue of the WCST (Robbins et al. 1998). Furthermore, the SWM and IED tasks engage the mid-dorsolateral/ventral PFC and the dorsolateral PFC respectively (Owen et al. 1995; Nagahama et al. 2001). As no group differences were identified on either of these tests, we found no evidence to support a general decrement in dorsolateral PFC-mediated cognitive function. Explanations for these disparate results may include the heterogeneity of IBS, differences in central functioning between IBS subtypes, temporal symptom fluctuations or subtle 
differences in the cognitive requirements for adequate performance on the IED and the WCST. Therefore, future studies exploring other aspects of executive function coupled with functional brain imaging are needed to clarify the impact, if any, on executive processes and other cognitive functions mediated by frontal brain regions in IBS.

In accordance with previous reports, levels of both depression and anxiety were higher in IBS (Hazlett-Stevens et al. 2003; Creed et al. 2005, 2008; Lembo et al. 2009; Mykletun et al. 2010) and depression was slightly elevated in CD (Goodhand et al. 2012). Cognitive deficits are well documented in depressive and anxiety disorders (Austin et al. 2001) and a relationship between the CAR and hippocampalmediated cognition in unmedicated depressed patients has been described recently (Hinkelmann et al. 2013). It is important to note that the cognitive deficits most clearly exhibited by patients with IBS (visuospatial episodic memory; PAL) and by patients with CD (Stroop interference) persisted after removal of patients exhibiting possible psychiatric co-morbidity, which strongly suggests that these deficits are independent of psychiatric co-morbidity. This result further suggests that a shared underlying pathophysiological mechanism, such has heightened levels of proinflammatory cytokines, which have been identified in both IBS (Dinan et al. 2006; Liebregts et al. 2007; Dinan et al. 2008; Scully et al. 2010; McKernan et al. 2011) and depression (Raison et al. 2006) and can impact on cognitive performance (Kennedy et al. 2012), may play a pivotal role in mediating the similarities in cognitive deficits identified in each disorder. Moreover, it is likely that similarities in cognitive dysfunction described here in IBS not only apply to depression but also overlap with other functional or psychiatric disorders in which stress, immune activation or altered central pain processing is implicated in the underlying pathophysiology. However, a note of caution is warranted in this interpretation as there is continuing debate in the literature as to the latent structure of the HADS and its utility in differentiating and detecting clinically relevant levels of anxiety and depression (Coyne \& van Sonderen, 2012a,b; Norton et al. 2012). Hence, future studies with a specific focus on identifying the shared and differential cognitive deficits associated with IBS and related psychiatric conditions are needed, with psychiatric status being formally determined using a SCID.

Of note, patients with CD exhibited a reduction in their capacity to cope with Stroop interference when compared to healthy control participants. They did not, however, exhibit visuospatial memory impairment. This suggests a disorder-specific mechanism by which HPA-axis dysfunction can impact neuro- biologically on cognitive performance. Cognitive function in IBD has not been well characterized, with only one study in the literature, lacking a healthy control comparison group, reporting a beneficial outcome of iron replacement therapy on cognitive performance in anaemic patients with CD (Wells et al. 2006). However, brain morphological abnormalities have recently been reported in CD (Agostini et al. 2013) and a subsequent review article has proposed that CD may be associated with altered cognitive performance due to these abnormalities (Vogt, 2013). Our results lend substantial support to this hypothesis and indicate that further investigations are needed at a structural and functional brain-imaging level to definitively link poor cognitive performance to the proposed morphological brain changes in $C D$.

A limitation of our study is the heterogeneous nature of our IBS population. There is some evidence to suggest that psychiatric diagnoses and possibly central functioning varies between IBS-D, IBS-C and IBS-A subtypes (Tillisch et al. 2005). Future studies, adequately powered to detect subgroup differences of cognitive performance in IBS, are needed, in addition to detecting potential differences in PAL performance between patients with $\mathrm{CD}$ and controls and patients with CD and IBS. In addition, the inclusion of patients with active and inactive $C D$ and ulcerative colitis, in conjunction with a more temporally specific measure of GI symptoms than the HBI, will be important in identifying whether cognitive differences exist between the two categories of IBD, due to disease activity and general GI symptomatology. Our results suggest that $\mathrm{CD}$ patients exhibit some differences in cognitive performance on the PAL and future studies should aim to explore this possibility in more detail. Whether the cognitive alterations identified here are state or trait cognitive markers requires further investigation by prospective and longitudinal study designs, particularly in relation to the potential impact of fluctuating symptoms and their severity on cognitive performance in IBS.

Our groups differed with regard to gender and it is important to note that gender differences in brain responses to visceral pain stimulation have been observed in IBS (Berman et al. 2000; Naliboff et al. 2003). Although small, and notably inconsistent, gender differences on visual-spatial tasks such as tests of spatial orientation or verbal abilities in healthy populations are commonly reported in the literature (Weiss et al. 2003), the influence of gender on visuospatial episodic memory performance in the PAL, to the best of our knowledge, has not been specifically investigated. It is therefore difficult to know whether gender differences impacted on our results, and future studies should aim to delineate the effects of gender on 
performance on the PAL and other assessments in IBS. Such studies will also need to examine the role that important factors such as age and menstrual cycle may play in cognitive performance in IBS.

In conclusion, IBS patients in the current study displayed a subtle but significant deficit on a hippocampal-mediated test of visuospatial episodic memory, which was related to morning cortisol levels and independent of psychiatric co-morbidity. Our results must be considered preliminary and this study as exploratory, and there is a clear need for follow-up studies to confirm these findings and to address the limitations discussed. Nonetheless, when considering that IBS is a common disorder and typically emerges in young adults who are involved in formal education or are in an early phase of career development, assessing the impact of IBS on cognition is of paramount importance. Daily stressors play a key role in exacerbating symptoms in IBS (Levy et al. 1997; Bennett et al. 1998) and our results suggest that, from a clinical point of view, therapies aimed at reducing stress or normalizing HPA-axis functioning should be considered a key line of treatment to reduce not only the impact on GI symptoms in IBS but also the central impact on cognitive function. Our results also have important implications for individuals who suffer from CD, which need to be addressed. Finally, in keeping with our primary hypothesis, these data support the view that IBS is a disorder associated with cognitive impairment (Kennedy et al. 2012) and warrant further investigation to identify the neurobiological mechanisms influencing cognitive performance in this debilitating disorder of the brain-gut axis.

\section{Acknowledgements}

We thank Dr C. O'Mahony and Dr A. Allen for their contributions to this study.

The Alimentary Pharmabiotic Centre is a research centre funded by Science Foundation Ireland (SFI), through the Irish Government's National Development Plan. We received support from the SFI (grant nos 02/CE/B124 and 07/CE/B1368) and the Health Research Board (HRB) through Health Research Awards (grant no. HRA_POR/2011/23; T.G.D., J.F.C. and G.C.). The Centre was previously funded by GlaxoSmithKline. J.F.C. is also funded by the European Community's Seventh Framework Programme; grant no.: FP7/2007-2013, grant agreement 201714. G.C. is in receipt of a research grant from the American Neurogastroenterology and Motility Society (ANMS). T.D., J.F.C., G.C. and E.M.M.Q. received support from the University College Cork (UCC) Strategic Research Fund towards the purchase of CANTAB software licenses. J.A.G. is funded by SFI
(09/RFP/NES2520), Ireland's Road Safety Authority, the UK Biotechnology and Biological Sciences Research Council (BBSRC, BB/F022883/1) and the US Air Force (AFOSR, FA-9550-08-1-0080).

\section{Declaration of Interest}

None.

\section{References}

Afzal M, Potokar JP, Probert CS, Munafo MR (2006). Selective processing of gastrointestinal symptom-related stimuli in irritable bowel syndrome. Psychosomatic Medicine 68, 758-761.

Agostini A, Benuzzi F, Filippini N, Bertani A, Scarcelli A, Farinelli V, Marchetta C, Calabrese C, Rizzello F, Gionchetti P, Ercolani M, Campieri M, Nichelli P (2013). New insights into the brain involvement in patients with Crohn's disease: a voxel-based morphometry study. Neurogastroenterology and Motility 25, 147-e82.

Aizawa E, Sato Y, Kochiyama T, Saito N, Izumiyama M, Morishita J, Kanazawa M, Shima K, Mushiake H, Hongo M, Fukudo S (2012). Altered cognitive function of prefrontal cortex during error feedback in patients with irritable bowel syndrome, based on FMRI and dynamic causal modeling. Gastroenterology 143, 1188-1198.

Alvarez JA, Emory E (2006). Executive function and the frontal lobes: a meta-analytic review. Neuropsychology Review 16, 17-42.

Assef EC, Capovilla AG, Capovilla FC (2007). Computerized Stroop test to assess selective attention in children with attention deficit hyperactivity disorder. Spanish Journal of Psychcology 10, 33-40.

Austin MP, Mitchell P, Goodwin GM (2001). Cognitive deficits in depression: possible implications for functional neuropathology. British Journal of Psychiatry 178, 200-206.

Barbara G, Stanghellini V, De Giorgio R, Cremon C, Cottrell GS, Santini D, Pasquinelli G, Morselli-Labate AM, Grady EF, Bunnett NW, Collins SM, Corinaldesi R (2004). Activated mast cells in proximity to colonic nerves correlate with abdominal pain in irritable bowel syndrome. Gastroenterology 126, 693-702.

Bender R, Lange S (2001). Adjusting for multiple testingwhen and how? Journal of Clinical Epidemiology 54, 343-349.

Bennett EJ, Tennant CC, Piesse C, Badcock CA, Kellow JE (1998). Level of chronic life stress predicts clinical outcome in irritable bowel syndrome. Gut 43, 256-261.

Berman S, Munakata J, Naliboff BD, Chang L, Mandelkern M, Silverman D, Kovalik E, Mayer EA (2000). Gender differences in regional brain response to visceral pressure in IBS patients. European Journal of Pain 4, 157-172.

Blackwell AD, Sahakian BJ, Vesey R, Semple JM, Robbins TW, Hodges JR (2004). Detecting dementia: novel neuropsychological markers of preclinical Alzheimer's disease. Dementia and Geriatric Cognitive Disorders 17, 42-48.

Bohmelt AH, Nater UM, Franke S, Hellhammer DH, Ehlert U (2005). Basal and stimulated 
hypothalamic-pituitary-adrenal axis activity in patients with functional gastrointestinal disorders and healthy controls. Psychosomatic Medicine 67, 288-294.

Botvinick MM, Cohen JD, Carter CS (2004). Conflict monitoring and anterior cingulate cortex: an update. Trends in Cognitive Sciences 8, 539-546.

Chang L, Sundaresh S, Elliott J, Anton PA, Baldi P, Licudine A, Mayer M, Vuong T, Hirano M, Naliboff BD, Ameen VZ, Mayer EA (2009). Dysregulation of the hypothalamic-pituitary-adrenal (HPA) axis in irritable bowel syndrome. Neurogastroenterology and Motility 21, 149-159.

Chapman S, Martin M (2011). Attention to pain words in irritable bowel syndrome: increased orienting and speeded engagement. British Journal of Health Psychology 16, 47-60.

Clarke G, Fitzgerald P, Cryan JF, Cassidy EM, Quigley EM, Dinan TG (2009a). Tryptophan degradation in irritable bowel syndrome: evidence of indoleamine 2,3-dioxygenase activation in a male cohort. BioMed Central

Gastroenterology 9, 6.

Clarke G, McKernan DP, Gaszner G, Quigley EM, Cryan JF, Dinan TG (2012). A distinct profile of tryptophan metabolism along the kynurenine pathway downstream of toll-like receptor activation in irritable bowel syndrome. Frontiers in Pharmacology 3, 90.

Clarke G, Quigley EMM, Cryan JF, Dinan TG (2009b). Irritable bowel syndrome: towards biomarker identification. Trends in Molecular Medicine 15, 478-489.

Collins SM (2005). Dysregulation of peripheral cytokine production in irritable bowel syndrome. American Journal of Gastroenterology 100, 2517-2518.

Collins SM, Surette M, Bercik P (2012). The interplay between the intestinal microbiota and the brain. Nature Reviews Microbiology 10, 735-742.

Coyne JC, van Sonderen E (2012a). The Hospital Anxiety and Depression Scale (HADS) is dead, but like Elvis, there will still be citings. Journal of Psychosomatic Research 73, 77-78.

Coyne JC, van Sonderen E (2012b). No further research needed: abandoning the Hospital and Anxiety Depression Scale (HADS). Journal of Psychosomatic Research 72, 173-174.

Creed F (2007). Cognitive behavioural model of irritable bowel syndrome. Gut 56, 1039-1041.

Creed F, Fernandes L, Guthrie E, Palmer S, Ratcliffe J, Read N, Rigby C, Thompson D, Tomenson B (2003). The cost-effectiveness of psychotherapy and paroxetine for severe irritable bowel syndrome. Gastroenterology 124, 303-317.

Creed F, Guthrie E, Ratcliffe J, Fernandes L, Rigby C, Tomenson B, Read N, Thompson DG (2005). Does psychological treatment help only those patients with severe irritable bowel syndrome who also have a concurrent psychiatric disorder? Australian and New Zealand Journal of Psychiatry 39, 807-815.

Creed F, Tomenson B, Guthrie E, Ratcliffe J, Fernandes L, Read N, Palmer S, Thompson DG (2008). The relationship between somatisation and outcome in patients with severe irritable bowel syndrome. Journal of Psychosomatic Research 64, 613-620.
Cryan JF, O'Mahony SM (2011). The microbiome-gut-brain axis: from bowel to behavior. Neurogastroenterology and Motility 23, 187-192.

de Kloet ER, Oitzl MS, Joels M (1999). Stress and cognition: are corticosteroids good or bad guys? Trends in Neurosciences 22, 422-426.

de Rover M, Pironti VA, McCabe JA, Acosta-Cabronero J, Arana FS, Morein-Zamir S, Hodges JR, Robbins TW, Fletcher PC, Nestor PJ, Sahakian BJ (2011). Hippocampal dysfunction in patients with mild cognitive impairment: a functional neuroimaging study of a visuospatial paired associates learning task. Neuropsychologia 49, 2060-2070.

Dinan TG, Clarke G, Quigley EM, Scott LV, Shanahan F, Cryan J, Cooney J, Keeling PW (2008). Enhanced cholinergic-mediated increase in the pro-inflammatory cytokine IL-6 in irritable bowel syndrome: role of muscarinic receptors. American Journal of Gastroenterology 103, 2570-2576.

Dinan TG, Quigley EM, Ahmed SM, Scully P, O'Brien S, O'Mahony L, O'Mahony S, Shanahan F, Keeling PW (2006). Hypothalamic-pituitary-gut axis dysregulation in irritable bowel syndrome: plasma cytokines as a potential biomarker? Gastroenterology 130, 304-311.

Downes JJ, Roberts AC, Sahakian BJ, Evenden JL, Morris RG, Robbins TW (1989). Impaired extra-dimensional shift performance in medicated and unmedicated Parkinson's disease: evidence for a specific attentional dysfunction. Neuropsychologia 27, 1329-1243.

Feise RJ (2002). Do multiple outcome measures require p-value adjustment? BMC Medical Research Methodology 2, 8 .

Fitzgerald P, Cassidy Eugene M, Clarke G, Scully P, Barry S, Quigley Eamonn MM, Shanahan F, Cryan J, Dinan Timothy G (2008). Tryptophan catabolism in females with irritable bowel syndrome: relationship to interferon-gamma, severity of symptoms and psychiatric co-morbidity. Neurogastroenterology and Motility 20, 1291-1297.

Ford AC, Forman D, Bailey AG, Axon AT, Moayyedi P (2008). Fluctuation of gastrointestinal symptoms in the community: a 10-year longitudinal follow-up study. Alimentary Pharmacology and Therapeutics 28, 1013-1020.

Fray PJ, Robbins TW (1996). CANTAB battery: proposed utility in neurotoxicology. Neurotoxicology and Teratology $\mathbf{1 8}$ 499-504.

Fries E, Dettenborn L, Kirschbaum C (2009). The cortisol awakening response (CAR): facts and future directions. International Journal of Psychophysiology 72, 67-73.

Gibbs-Gallagher N, Palsson OS, Levy RL, Meyer K, Drossman DA, Whitehead WE (2001). Selective recall of gastrointestinal-sensation words: evidence for a cognitive-behavioral contribution to irritable bowel syndrome. American Journal of Gastroenterology 96, 1133-1138.

Glass JM (2006). Cognitive dysfunction in fibromyalgia and chronic fatigue syndrome: new trends and future directions. Current Rheumatology Reports 8, 425-429.

Gomborone JE, Dewsnap PA, Libby GW, Farthing MJ (1993). Selective affective biasing in recognition memory in the irritable bowel syndrome. Gut 34, 1230-1233. 
Goodhand JR, Wahed M, Mawdsley JE, Farmer AD, Aziz Q, Rampton DS (2012). Mood disorders in inflammatory bowel disease: relation to diagnosis, disease activity, perceived stress, and other factors. Inflammatory Bowel Diseases 18, 2301-2309.

Grenham S, Clarke G, Cryan JF, Dinan TG (2011). Brain-gut-microbe communication in health and disease. Frontiers in Physiology 2, 94.

Guilarte M, Santos J, de Torres I, Alonso C, Vicario M, Ramos L, Martinez C, Casellas F, Saperas E, Malagelada JR (2007). Diarrhoea-predominant IBS patients show mast cell activation and hyperplasia in the jejunum. Gut 56, 203-209.

Hazlett-Stevens H, Craske MG, Mayer EA, Chang L, Naliboff BD (2003). Prevalence of irritable bowel syndrome among university students: the roles of worry, neuroticism, anxiety sensitivity and visceral anxiety. Journal of Psychosomatic Research 55, 501-505.

Hinkelmann K, Muhtz C, Dettenborn L, Agorastos A, Moritz S, Wingenfeld K, Spitzer C, Gold SM, Wiedemann K, Otte C (2013). Association between cortisol awakening response and memory function in major depression. Psychological Medicine. Published online: 27 February 2013. doi:10.1017/S0033291713000287.

Kennedy PJ, Clarke G, Quigley EM, Groeger JA, Dinan TG, Cryan JF (2012). Gut memories: towards a cognitive neurobiology of irritable bowel syndrome. Neuroscience and Biobehavioral Reviews 36, 310-340.

Kroenke K, Spitzer RL, Williams JB (2001). The PHQ-9: validity of a brief depression severity measure. Journal of General Internal Medicine 16, 606-613.

Lackner JM, Lou Coad M, Mertz HR, Wack DS, Katz LA, Krasner SS, Firth R, Mahl TC, Lockwood AH (2006). Cognitive therapy for irritable bowel syndrome is associated with reduced limbic activity, GI symptoms, and anxiety. Behaviour Research and Therapy 44, 621-638.

Law R, Hucklebridge F, Thorn L, Evans P, Clow A (2013). State variation in the cortisol awakening response. Stress. Published online: 29 July 2013. doi:10.3109/ 10253890.2013.817552.

Lembo AJ, Zaman M, Krueger RF, Tomenson BM, Creed FH (2009). Psychiatric disorder, irritable bowel syndrome, and extra-intestinal symptoms in a population-based sample of twins. American Journal of Gastroenterology 104, 686-694.

Levy RL, Cain KC, Jarrett M, Heitkemper MM (1997). The relationship between daily life stress and gastrointestinal symptoms in women with irritable bowel syndrome. Journal of Behavioral Medicine 20, 177-193.

Liebregts T, Adam B, Bredack C, Roth A, Heinzel S, Lester S, Downie-Doyle S, Smith E, Drew P, Talley NJ, Holtmann G (2007). Immune activation in patients with irritable bowel syndrome. Gastroenterology 132, 913-920.

Longstreth GF, Thompson WG, Chey WD, Houghton LA, Mearin F, Spiller RC (2006). Functional bowel disorders. Gastroenterology 130, 1480-1491.

Longstreth GF, Wilson A, Knight K, Wong J, Chiou CF, Barghout V, Frech F, Ofman JJ (2003). Irritable bowel syndrome, health care use, and costs: a U.S. managed care perspective. American Journal of Gastroenterology 98, 600-607.
Lupien SJ, Maheu F, Tu M, Fiocco A, Schramek TE (2007). The effects of stress and stress hormones on human cognition: implications for the field of brain and cognition. Brain and Cognition 65, 209-237.

Lupien SJ, McEwen BS, Gunnar MR, Heim C (2009). Effects of stress throughout the lifespan on the brain, behaviour and cognition. Nature Reviews Neuroscience 10, 434-445.

Martin M, Chapman SC (2010). Cognitive processing in putative functional gastrointestinal disorder: rumination yields orientation to social threat not pain. European Journal of Pain 14, 207-213.

Mayer EA (2000). The neurobiology of stress and gastrointestinal disease. Gut 47, 861-869.

Mayer EA, Aziz Q, Coen S, Kern M, Labus JS, Lane R, Kuo B, Naliboff B, Tracey I (2009). Brain imaging approaches to the study of functional GI disorders: a Rome working team report. Neurogastroenterology and Motility 21, 579-596.

Mayer EA, Naliboff BD, Chang L, Coutinho SV (2001). V. Stress and irritable bowel syndrome. American Journal of Physiology Gastrointestinal and Liver Physiology 280, G519-G524.

Mayer EA, Tillisch K (2011). The brain-gut axis in abdominal pain syndromes. Annual Review of Medicine 62, 381-396.

McEwen BS (1999). Stress and the aging hippocampus. Frontiers in Neuroendocrinology 20, 49-70.

McKernan DP, Gaszner G, Quigley EM, Cryan JF, Dinan TG (2011). Altered peripheral toll-like receptor responses in the irritable bowel syndrome. Alimentary Pharmacology and Therapeutics 33, 1045-1052.

Mehta MA, Sahakian BJ, McKenna PJ, Robbins TW (1999). Systemic sulpiride in young adult volunteers simulates the profile of cognitive deficits in Parkinson's disease. Psychopharmacology 146, 162-174.

Moriarty O, McGuire BE, Finn DP (2011). The effect of pain on cognitive function: a review of clinical and preclinical research. Progress in Neurobiology 93, 385-404.

Mykletun A, Jacka F, Williams L, Pasco J, Henry M, Nicholson GC, Kotowicz MA, Berk M (2010). Prevalence of mood and anxiety disorder in self reported irritable bowel syndrome (IBS). An epidemiological population based study of women. BioMed Central Gastroenterology 10, 88 .

Nagahama Y, Okada T, Katsumi Y, Hayashi T, Yamauchi H, Oyanagi C, Konishi J, Fukuyama H, Shibasaki H (2001). Dissociable mechanisms of attentional control within the human prefrontal cortex. Cerebral Cortex 11, 85-92.

Naliboff BD, Berman S, Chang L, Derbyshire SW, Suyenobu B, Vogt BA, Mandelkern M, Mayer EA (2003). Sex-related differences in IBS patients: central processing of visceral stimuli. Gastroenterology 124, 1738-1747.

Nelson H, Willison J (1991). National Adult Reading Test (NART): Test Manual. NFER Nelson: Windsor.

Norton S, Sacker A, Done J (2012). Further research needed: a comment on Coyne and van Sonderen's call to abandon the Hospital Anxiety and Depression Scale. Journal of Psychosomatic Research 73, 75-76; author reply 77-78. 
Ohman L, Simren M (2007). New insights into the pathogenesis and pathophysiology of irritable bowel syndrome. Digestive and Liver Disease 39, 201-215.

Ohman L, Simren M (2010). Pathogenesis of IBS: role of inflammation, immunity and neuroimmune interactions. Nature Reviews Gastroenterology and Hepatology 7, 163-173.

Owen AM, Morris RG, Sahakian BJ, Polkey CE, Robbins TW (1996). Double dissociations of memory and executive functions in working memory tasks following frontal lobe excisions, temporal lobe excisions or amygdalo-hippocampectomy in man. Brain 119, 1597-1615.

Owen AM, Sahakian BJ, Semple J, Polkey CE, Robbins TW (1995). Visuo-spatial short-term recognition memory and learning after temporal lobe excisions, frontal lobe excisions or amygdalo-hippocampectomy in man. Neuropsychologia 33, 1-24.

Posserud I, Svedlund J, Wallin J, Simren M (2009). Hypervigilance in irritable bowel syndrome compared with organic gastrointestinal disease. Journal of Psychosomatic Research 66, 399-405.

Pruessner JC, Kirschbaum C, Meinlschmid G, Hellhammer DH (2003). Two formulas for computation of the area under the curve represent measures of total hormone concentration versus time-dependent change. Psychoneuroendocrinology 28, 916-931.

Quigley EM (2006). Changing face of irritable bowel syndrome. World Journal of Gastroenterology 12, 1-5.

Rahman S, Sahakian BJ, Hodges JR, Rogers RD, Robbins TW (1999). Specific cognitive deficits in mild frontal variant frontotemporal dementia. Brain 122, 1469-1493.

Raison CL, Capuron L, Miller AH (2006). Cytokines sing the blues: inflammation and the pathogenesis of depression.

Trends in Immunology 27, 24-31.

Robbins TW, James M, Owen AM, Sahakian BJ, Lawrence AD, McInnes L, Rabbitt PM (1998). A study of performance on tests from the CANTAB battery sensitive to frontal lobe dysfunction in a large sample of normal volunteers: implications for theories of executive functioning and cognitive aging. Cambridge Neuropsychological Test Automated Battery. Journal of the International Neuropsychological Society 4, 474-490.

Robbins TW, Sahakian BJ (1994). Computer methods of assessment of cognitive function. In Principles and Practice of Geriatric Psychiatry, pp. 205-209. John Wiley \& Sons Ltd: Chichester.

Sahakian BJ, Owen AM (1992). Computerized assessment in neuropsychiatry using CANTAB: discussion paper. Journal of the Royal Society of Medicine 85, 399-402.

Scully P, McKernan DP, Keohane J, Groeger D, Shanahan F, Dinan TG, Quigley EM (2010). Plasma cytokine profiles in females with irritable bowel syndrome and extra-intestinal co-morbidity. American Journal of Gastroenterology 10, 2235-2243.

Sharpe M, Peveler R, Mayou R (1992). The psychological treatment of patients with functional somatic symptoms: a practical guide. Journal of Psychosomatic Research 36, 515-529.
Snaith RP (2003). The Hospital Anxiety and Depression Scale. Health and Quality of Life Outcomes 1, 29.

Song L, Che W, Min-Wei W, Murakami Y, Matsumoto K (2006). Impairment of the spatial learning and memory induced by learned helplessness and chronic mild stress. Pharmacology, Biochemistry and Behavior 83, 186-193.

Spence MJ, Moss-Morris R (2007). The cognitive behavioural model of irritable bowel syndrome: a prospective investigation of patients with gastroenteritis. Gut 56, 1066-1071.

Spiegel BM (2009). The burden of IBS: looking at metrics. Current Gastroenterology Reports 11, 265-269.

Sterlemann V, Rammes G, Wolf M, Liebl C, Ganea K, Muller MB, Schmidt MV (2010). Chronic social stress during adolescence induces cognitive impairment in aged mice. Hippocampus 20, 540-549.

Strauss E, Sherman EMS, Spreen O (2006). Compendium of Neuropsychological Tests: Administration, Norms, and Commentary. Oxford University Press: New York.

Suarez-Hitz KA, Otto B, Bidlingmaier M, Schwizer W, Fried M, Ehlert U (2012). Altered psychobiological responsiveness in women with irritable bowel syndrome. Psychosomatic Medicine 74, 221-231.

Swainson R, Hodges JR, Galton CJ, Semple J, Michael A, Dunn BD, Iddon JL, Robbins TW, Sahakian BJ (2001). Early detection and differential diagnosis of Alzheimer's disease and depression with neuropsychological tasks. Dementia and Geriatric Cognitive Disorders 12, 265-280.

Sweeney JA, Kmiec JA, Kupfer DJ (2000). Neuropsychologic impairments in bipolar and unipolar mood disorders on the CANTAB neurocognitive battery. Biological Psychiatry 48, 674-684.

Tillisch K, Labus JS, Naliboff BD, Bolus R, Shetzline M, Mayer EA, Chang L (2005). Characterization of the alternating bowel habit subtype in patients with irritable bowel syndrome. American Journal of Gastroenterology 100, 896-904.

Tillisch K, Mayer EA, Labus JS (2011). Quantitative meta-analysis identifies brain regions activated during rectal distension in irritable bowel syndrome. Gastroenterology 140, 91-100.

Vogt BA (2013). Inflammatory bowel disease: perspectives from cingulate cortex in the first brain. Neurogastroenterology and Motility 25, 93-98.

Weiss EM, Kemmler G, Deisenhammer EA, Fleischhacker WW, Delazer M (2003). Sex differences in cognitive functions. Personality and Individual Differences 35, 863-875.

Wells CW, Lewis S, Barton JR, Corbett S (2006). Effects of changes in hemoglobin level on quality of life and cognitive function in inflammatory bowel disease patients. Inflammatory Bowel Diseases 12, 123-130.

Whorwell PJ, Prior A, Colgan SM (1987). Hypnotherapy in severe irritable bowel syndrome: further experience. Gut $\mathbf{2 8}$, 423-425.

Whorwell PJ, Prior A, Faragher EB (1984). Controlled trial of hypnotherapy in the treatment of 
severe refractory irritable-bowel syndrome. Lancet 2, 1232-1234.

Wilson S, Roberts L, Roalfe A, Bridge P, Singh S (2004). Prevalence of irritable bowel syndrome: a community survey. British Journal of Gerneral Practice 54, 495-502.
Wolf OT (2003). HPA axis and memory. Best Practice and Research: Clinical Endocrinology and Metabolism 17, 287-299.

Zigmond AS, Snaith RP (1983). The hospital anxiety and depression scale. Acta Psychiatrica Scandinavica 67, 361-370. 